\title{
Large Carnivore Attacks on Humans: The State of Knowledge
}

\author{
Jennifer Rebecca Kelly' \\ Environmental Studies Program, Boston College \\ Massachusetts, United States of America \\ Thomas J. Doherty \\ Sustainable Self \\ Portland, Oregon, United States of America \\ Thomas Gabel \\ Environmental Studies Program, Boston College \\ Massachusetts, United States of America \\ Willa Disbrow \\ Environmental Studies Program, Boston College \\ Massachusetts, United States of America
}

\section{Abstract}

In this paper, we summarize the state of the literature regarding attacks on humans from large carnivores, and classify them, where possible, according to three common precursors of such attacks including human provocation and animal disease. We found the risk of a large carnivore attacking a human is relatively low in comparison to other natural threats, such as being struck by lightning. Our recommendations include ways for humans to coexist with large carnivores, such as aversive conditioning of habituated carnivores. Finally, we argue for a more standardized method of obtaining attack information across scholars and practitioners such as the use of consistent timelines, regions and sources, the inclusion of gray literature, and the recording of causal factors such as provocation and disease. Empirical knowledge of carnivore attacks can augment and inform individual and culturally influenced understandings with the potential for more humane, effective, and locally appropriate wildlife management and conservation techniques.

Keywords: attacks, human-wildlife interactions, large carnivores, predators

1 Corresponding author: jennifer.kelly.3@bc.edu 


\section{Introduction}

Representations in popular discourse of attacks on humans by large carnivores often assume the wild animal is in some way to "blame" for the attack. However, the circumstances of wild animal attacks on humans are multilayered. Apart from unprovoked attacks (e.g., predation), common precursors to large carnivore attacks are human behaviors and practices.

In this paper, we summarize the state of the literature regarding attacks on humans by large carnivores. In doing so, we have two main objectives: (1) to quantify such attacks in a single record with a view to providing accurate accounting, and classify the causal factors as much as possible as part of that account; and (2) to assist future research into the development of more systematic methods and criteria for reporting of and compiling data on such attacks, by providing scholars and practitioners with a reference paper on the range of existing methods. We extend these objectives by using the numbers compiled to illuminate the relatively low risk of carnivore attack compared to other threats to human safety from the natural world, and to argue for more thorough and systematic record-keeping of attacks. Furthermore, aligning with a shift in public sentiment away from lethal control of large carnivores, we recommend some ways to coexist with wild animals and reduce the incidence of attacks.

Many factors can contribute to wild carnivore attacks on humans. Large carnivores may, unprovoked, predate on humans as they would with other prey animals, but provocations (intentional and unintentional) from human behaviors and practices can also contribute to encounters that result in attacks. Apart from human behaviors intended to harm, antagonize, or threaten an animal or its young, unintentional human provocations such as food-conditioning and other forms of habituation of an animal may be a precursor to an attack. For example, failure to understand proper food storage when camping in bear habitat can result in an encounter with a bear (which, while unintended, would be considered a provocation if followed by an attack). Habituation describes the process by which an individual wild animal becomes accustomed to the presence of humans. Food-conditioning, while a form of habituation, is distinct (Smith et al., 2005, p. 2) in that it describes a wild animal who feeds on human food (e.g., direct feeding by humans, taking food from campsites, or eating human garbage). In addition to considerations of provocation, Quigley and Herrero (2005) describe yet another layer of attacks: that of rabid or diseased animals, as disease may disrupt an animal's normal behaviors, which could result in an attack. Despite the complexities of wild animal attacks on humans, these common factors-provocation (including unintended forms such as habituation) and disease - are not widely discussed in the literature in such a way as to distinguish them from unprovoked attacks. 
In our research we encountered considerable inconsistency in the available literature in the reporting and recording of information on wild animal attacks on humans. First, the terminology and framing used to report on attacks varied based on the objective or type of the source (newspaper, academic journal, database, etc.). Second, animal attack information on human injuries and fatalities was not consistent across species, time, and geopolitical boundaries. Third, some sources described and classified the same attack in different ways. Finally, the method of data collection and time periods covered varied across sources.

\section{Methods}

We conducted a literature search of large carnivore attacks on humans through Google Scholar. The sources included published media reports, interviews and surveys with victims or witnesses of attacks, and information obtained from nationally or academically maintained databases. We used primarily quantitative data rather than qualitative data, although some of the sources we used analyzed news reports. We did not use case studies as the sample size was too small to produce meaningful results. From articles published between 1982 and 2012 we selected those that had been cited more frequently, ranging from 20 to 220 citations. For more recent articles, 2013 to 2019, we did not consider the number of citations given that citation rates for these are still growing. Where sufficient information was available, we identified characteristics of the attacks as they were described in the source, including where the source itself had provided a classification. We then classified the instances into four categories: Provoked, Unprovoked, Diseased, and Unclassified. We followed the work of Quigley and Herrero (2005) to determine parameters for extending and refining our definitions of Provoked and Unprovoked attacks. Table 1 draws from these established definitions for Provoked and Unprovoked in the literature, and to give a sense of the various ways such concepts are used.

The classification Diseased was chosen where the information in the source was sufficient to identify a diseased animal. Few of the sources noted the overall condition of the animal beyond identifiable diseases such as rabies. Moreover, the data available were insufficient to capture other causes of poor health in an animal, such as injury or malnourishment, which are also likely causal factors in attacks. For example, Herrero et al.'s (2011) study of black bear attacks reported that 68\% of fatalities (23 of 34) were associated with bears exhibiting signs of poor health such as "underweight, injured, poor condition" (p. 599). Where the details of attacks were explicit, we were able to classify some of these as Diseased. 


\section{Table 1. Definitions and descriptions of Provoked and Unprovoked attacks}

\begin{tabular}{|c|c|c|}
\hline Species* & $\begin{array}{l}\text { Provoked } \\
\text { Person(s) enters an animal's personal } \\
\text { space or purposely tries to touch, injure } \\
\text { or kill the animal, and the animal attacks } \\
\text { (Quigley \& Herrero, 2005). }\end{array}$ & $\begin{array}{l}\text { Unprovoked } \\
\text { Animal approaches and attacks, with the } \\
\text { principal attraction being the person(s) } \\
\text { (Quigley \& Herrero, 2005). }\end{array}$ \\
\hline $\begin{array}{l}\text { Coyote } \\
\text { (Canis latrans) }\end{array}$ & $\begin{array}{l}\text { Defensive: Person(s) corners coyote, } \\
\text { or coyote is defending pups or a den. } \\
\text { Companion animal** related: Person } \\
\text { is bitten while a domestic companion } \\
\text { animal is present (White \& Gehrt, 2009). }\end{array}$ & $\begin{array}{l}\text { Predatory: Coyote directly and } \\
\text { aggressively pursues and bites a victim, } \\
\text { including attacks from pairs and packs. } \\
\text { Investigative: Coyote tests the victim as } \\
\text { possible prey, victim is sleeping or resting } \\
\text { when bitten (White \& Gehrt, 2009). }\end{array}$ \\
\hline Shark & $\begin{array}{l}\text { A person initiates contact with a shark, } \\
\text { is spearfishing or feeding sharks, or is } \\
\text { unhooking a shark from a fishing net } \\
\text { (Naylor \& Bowling, 2018). }\end{array}$ & $\begin{array}{l}\text { Attack on a human occurs in the shark's } \\
\text { natural habitat with no provocation of the } \\
\text { shark (Naylor \& Bowling, 2018). }\end{array}$ \\
\hline Bear & $\begin{array}{l}\text { Person is perceived by the bear as } \\
\text { a threat, or comes between a sow and } \\
\text { her cubs. }\end{array}$ & $\begin{array}{l}\text { Person does not threaten the bear } \\
\text { and/or is not in the presence of food. } \\
\text { Instances where a sleeping human is } \\
\text { dragged by a bear and eaten. }\end{array}$ \\
\hline $\begin{array}{l}\text { Wolf } \\
\text { (Canis lupus) }\end{array}$ & $\begin{array}{l}\text { Defensive: Defending dens, rendezvous } \\
\text { sites, or disabled conspecifics. } \\
\text { Provoked aggression in self-defense: } \\
\text { Aggression in response to pursuit by } \\
\text { a human. } \\
\text { Agonistic: Animal exhibits aggression } \\
\text { or avoidance. These include: person's } \\
\text { activity is threatening, annoying } \\
\text { or unexpected, instances of food- } \\
\text { conditioning, or person is accompanied } \\
\text { by dogs (McNay, 2002). }\end{array}$ & $\begin{array}{l}\text { Predatory: Wolf establishes orientation } \\
\text { toward the prey, stalking, chasing, biting. } \\
\text { Prey-testing agonistic charges: } \\
\text { Aggressive charges or leaps toward } \\
\text { people but the motivations are unclear. } \\
\text { These could include: a misidentification } \\
\text { of people as prey, naive wolves testing } \\
\text { unfamiliar prey, wolves feeling compelled } \\
\text { to aggressively drive people away, or } \\
\text { non-habituated wolves (McNay, 2002). }\end{array}$ \\
\hline
\end{tabular}

* Scientific name included only where citation has done so.

** "Pet" is the common term used in the human-wildlife literature, however, we use companion animal, which has recently emerged in the animal studies literature to minimize the role of domination.

Where the source contained insufficient information to classify an attack as Provoked, Unprovoked, or Diseased, the fourth category of Unclassified was used.

We recognize as limitations in our research the inconsistencies noted above in reporting and record-keeping of these data. However, the two main objectives of our study_compiling attack numbers as thoroughly as possible, and summarizing the state of record-keeping methods and criteria-justified gathering our data from the widest possible range of sources, time spans, and land areas. Given the existing inconsistencies in the source literature, narrowing our focus to only the most comparable sources would have omitted most of the available data on attacks, and failed to adequately address the current state of data available on this topic. 


\section{Results}

Table 2 provides the data from our search: location, date range, and frequency of attacks on humans (including human fatalities, where known) from large carnivorous species. As discussed above, we identified attacks as Provoked, Unprovoked, Diseased, or Unclassified (where sources provided insufficient detail for classification). We list global data on human attacks from 17 species of large carnivores. Polar bears had the longest recorded data at 144 years, during which time 73 attacks took place globally (Wilder et al., 2017). Sharks had the highest frequency of attacks at 984 over an 11-year period. Leopards had the second-highest frequency of recorded attacks at 840 across a 100-year time span in India, Nepal, Uganda, and South Africa (Löe \& Röskaft, 2004). Cheetahs and snow leopards had no recorded attacks globally in studies up to 2009 (Inskip \& Zimmerman, 2009).

Details for those species for which the literature met one or more of the following criteria: (1) recorded a long time range of collected data, (2) covered a large geographic area, (3) provided unique insight, or (4) described categories consistent with our Provoked or Unprovoked classifications, are discussed below.

\section{Black bears (Ursus americanus)}

From 1900 to 2009 there were a total of 60 attacks on humans by black bears resulting in 63 deaths $^{2}$ in the United States and Canada (Herrero et al., 2011). Non-fatal outcomes were not reported in this study. Of these attacks, 49 were Unprovoked, defined as natural predatory behavior of a bear (Herrero et al., 2011). We were unable to record the provoked attacks clearly with the information provided. For example, Herrero et al. (2011) found 15 fatalities involved human food or garbage, one involved a hunter wounding a bear, and two involved a bear breaking into a cabin. Furthermore, 11 were considered to have a health problem (Diseased), but it was not clear if these 11 bears had been provoked or were unprovoked by humans. These data were procured through the national park services of each state, territory, or province in the United States and Canada, as well as electronic newspaper reports (Herrero et al., 2011).

In British Columbia, Canada, there were 22 attacks by black bears on humans from 1960 to 1997 (Herrero \& Higgins, 1999). Data for these attacks were obtained through a scientific literature review, as well as by contacting all wildlife management agencies operating within British Columbia during this time period. Of these attacks, 18 were Unprovoked or predatory in nature (Herrero \& Higgins, 1999). In three instances, the bear was reported by wildlife officials to have a "low body weight" (Diseased) (Herrero \& Higgins, 1999, p. 214).

2 In some cases, more than one person was attacked in the incident. 


\section{Brown bears (Ursus arctos)}

Using wildlife management agency records, newspapers, and a bear attack database, Smith (2005) found 420 brown bear incidents with humans in Alaska between 1990 and 2004. Of these, 216 attacks were described in the source as "aggressivedefensive" and the remaining incidents were "attributed to curiosity, attraction to foodstuffs, carcass defense, or provocation" (Smith et al., 2005, p. 2), but could not be clearly classified as Provoked, or even as an attack. All "aggressive-defensive" attacks were Unclassified as there was no clarification whether the attack was aggressive (i.e., unprovoked) or defensive (i.e., provoked).

Through a scientific literature review, as well as in gathering data from wildlife management agencies operating within British Columbia, Canada, Herrero and Higgins (1999) found 49 brown bears had attacked humans from 1960 to 1997. Of the 49, we classified five attacks as Unprovoked, three were recorded by wildlife officials of animals in "poor physical condition" (Diseased) (Herrero \& Higgins, 1999, p. 214), and the 41 remaining incidents as Unclassified.

\section{Polar bears (Ursus maritimus)}

From 2005 to 2016, there was a total of 68 Unprovoked polar bear attacks reported in the media globally (Bombieri, 2018). In North America, Europe (Svalbard, Norway), and Russia there were 13 attacks on humans from 1955 to 2014 (Penteriani et al., 2016). These findings were obtained from Wikipedia and online newspapers, where attacks were reported with insufficient detail to determine classification (Unclassified). In a global study from 1870 to 2014, Wilder et al. (2017) reported 73 attacks by polar bears on humans. Of these, 20 people were killed and 63 people were injured. ${ }^{3}$ By region, 38 occurred in Canada, four in Greenland, 10 in Norway, 15 in Russia, and six in the United States (Wilder et al., 2017, p. 540). The study defined attacks as "predatory" in instances where the bear made "intentional contact in human injury ... preyed upon, or attempted to prey upon, people ... [or were involved in] behavioral components such as stalking and rushing the victim" (Wilder et al., 2017, p. 539). Based on this definition, we identified 37 Unprovoked attacks on humans. Of the 30 Provoked attacks, 14 were attributed to anthropogenic attractants such as "garbage, harvested animals, meat caches, and dog yards" (Wilder et al., 2017, p. 544).

3 In some cases, more than one person was attacked in the incident. 


\section{Sloth bears (Melursus ursinus)}

In Sri Lanka, interviews with victims and eyewitnesses revealed 271 attacks on humans from 1938 to 2004 (Ratnayeke et al., 2014). Provoked attacks included 82 reports of bear cubs being present, three instances where the bear was provoked by a dog, three cases where a human tried to drive a bear off its food source, and two encounters which resulted in a human shooting at the bear "to avert a possible attack" (Ratnayeke et al., 2014, p. 472). Eleven involved cases where a person was run down by a bear (Ratnayeke et al., 2014), but it was unclear if the attack was provoked so we placed these 11 with the remaining attacks that could not be confidently classified (Unclassified).

\section{Coyotes (Canis latrans)}

White and Gehrt (2009) analyzed databases, newspapers, and Google News archives to report 142 coyote attacks on 159 victims in urban areas in the United States and Canada from 1960 to 2006. Of these, 63 were described as predatory and 32 were investigative, classified here as 95 Unprovoked attacks. A total of 13 Provoked attacks included eight related to companion animals, and five in which coyotes were defending themselves, cubs, or a den. A further 15 were classified as rabid coyotes (Diseased) and 36 were unknown cases (Unclassified).

\section{Wolves (Canis lupus)}

McNay (2002) reviewed cases from biologists, locals, law officials, public health records, and published literature in order to identify 50 aggressive encounters with wolves in Alaska, Minnesota's Canadian border, and Canada from 1969 to 2000. It was reported that healthy wolves were responsible for 38 of these attacks, while 12 were rabid (Diseased). In six cases where healthy wolves attacked, dogs were present (Provoked). Of the 18 Unprovoked attacks, none were life-threatening, although 13 ended in a bite, four of which were severe (McNay, 2002, Table 2, p. 835).

\section{Leopards (Panthera pardus)}

According to the Ugandan Government, there were 114 attacks by leopards on humans in Uganda from 1923 to 1994 (Treves \& Naughton-Treves, 1999), where $32.5 \%$ of these resulted in human fatalities. Of the people killed by leopards, $75 \%$ were women or children. Of the 114 attacks, four were a result of leopards protecting their kill from humans (Provoked) (Treves \& Naughton-Treves, 1999). 


\section{Lions (Panthera leo)}

In Rufiji and Lindi, Tanzania, from 1990 to 2007, there were 274 attacks on humans (Kushnir, 2009). Of these, all (99 in Rufiji and 175 in Lindi) were Unprovoked (Kushnir, 2009), attributed to predation. High concentrations of bush pigs (Potamochoerus porcus) and low numbers of other forms of prey in both districts was correlated with a higher frequency of lion attacks (Kushnir, 2009). Lion attack information was procured from the District Game Office and follow-up interviews with “survivors, victims' family members, or village leaders" (Kushnir, 2009, p. 6).

In Uganda, drawing on data maintained by the Ugandan Government, there were 275 attacks by lions on humans in Uganda from 1923 to 1994 (Treves \& NaughtonTreves, 1999). Of these, $74.9 \%$ of attacks were fatal. Five of the 275 attacks were Provoked, where lions were attempting to defend their kills from human scavengers (Treves \& Naughton-Treves, 1999).

\section{Sharks}

According to the International Shark Attack File, there has been a total of 984 attacks by sharks on humans from 2007 to 2018 (ISAF, 2018). Of these attacks, great white (or white pointer) sharks (Carcharodon carcharias), tiger sharks (Galeocerdo cuvier), and bull sharks (Carcharhinus leucas) were responsible for 314, 111, and 100 attacks on humans, respectively.

In 2018, of a global total of 130 attacks reported, there were 66 Unprovoked and 34 Provoked attacks. The remaining 30 attacks we grouped as Unclassified. These comprised a mix of attacks on boats, post-mortem bites, unverified and doubtful reports (including where the attacking animal may not have been a shark), among others (Naylor \& Bowling, 2018). 
Table 2. Large carnivore attacks on humans

\begin{tabular}{|c|c|c|c|c|c|c|c|}
\hline & $\begin{array}{l}\text { Date } \\
\text { range }\end{array}$ & Location (Source) & 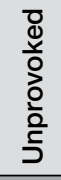 & $\begin{array}{l}\text { రृ } \\
\text { ํ. } \\
\text { ō } \\
\text { ò }\end{array}$ & 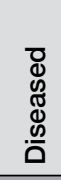 & 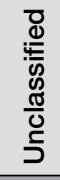 & $\begin{array}{l}\text { Total attacks } \\
\text { (per date range) }\end{array}$ \\
\hline \multicolumn{8}{|l|}{ Bears (Ursidae) } \\
\hline \multirow{5}{*}{$\begin{array}{l}\text { Black bear } \\
\text { (Ursus } \\
\text { americanus) }\end{array}$} & $\begin{array}{l}1900- \\
2009 \\
\end{array}$ & $\begin{array}{l}\text { United States \& Canada } \\
\text { (Herrero et al., 2011) }\end{array}$ & 49 & NR & 11 & $N R$ & $\begin{array}{l}63^{\star} \\
\text { (109 years) }\end{array}$ \\
\hline & $\begin{array}{l}1900- \\
2000\end{array}$ & $\begin{array}{l}\text { United States \& Canada } \\
\text { (Löe \& Röskaft, 2004) }\end{array}$ & $N R$ & NR & $N R$ & 37 & $\begin{array}{l}37 \\
(100 \text { years) }\end{array}$ \\
\hline & $\begin{array}{l}1955- \\
2014 \\
\end{array}$ & $\begin{array}{l}\text { North America } \\
\text { (Penteriani et al., 2016) }\end{array}$ & NR & NR & $\mathrm{NR}$ & 85 & $\begin{array}{l}85 \\
(59 \text { years }) \\
\end{array}$ \\
\hline & $\begin{array}{l}1960- \\
1997 \\
\end{array}$ & $\begin{array}{l}\text { British Columbia, Canada } \\
\text { (Herrero \& Higgins, 1999) }\end{array}$ & 18 & NR & 3 & 1 & $\begin{array}{l}22 \\
\text { (37 years) }\end{array}$ \\
\hline & $\begin{array}{l}2005- \\
2016 \\
\end{array}$ & $\begin{array}{l}\text { Global } \\
\text { (Bombieri et al., 2018) }\end{array}$ & $N R$ & $N R$ & NR & 164 & $\begin{array}{l}164 \\
(11 \text { years })\end{array}$ \\
\hline \multirow[t]{7}{*}{$\begin{array}{l}\text { Brown bear } \\
\text { (Ursus arctos) }\end{array}$} & $\begin{array}{l}1900- \\
2000 \\
\end{array}$ & $\begin{array}{l}\text { Global\# } \\
\text { (Löe \& Röskaft, 2004) } \\
\end{array}$ & $N R$ & NR & $N R$ & 313 & $\begin{array}{l}313^{*} \\
(100 \text { years }) \\
\end{array}$ \\
\hline & $\begin{array}{l}1955- \\
2014 \\
\end{array}$ & $\begin{array}{l}\text { North America } \\
\text { (Penteriani et al., 2016) } \\
\end{array}$ & NR & NR & NR & 92 & \begin{tabular}{|l}
92 \\
$(59$ years $)$ \\
\end{tabular} \\
\hline & $\begin{array}{l}1960- \\
1997 \\
\end{array}$ & $\begin{array}{l}\text { British Columbia, Canada } \\
\text { (Herrero \& Higgins, 1999) }\end{array}$ & 5 & NR & 3 & 41 & $\begin{array}{l}49 \\
\text { (37 years) } \\
\end{array}$ \\
\hline & $\begin{array}{l}1955- \\
2014 \\
\end{array}$ & $\begin{array}{l}\text { Sweden, Finland \& Spain } \\
\text { (Penteriani et al., 2016) }\end{array}$ & $N R$ & NR & NR & 65 & $\begin{array}{l}65 \\
(59 \text { years }) \\
\end{array}$ \\
\hline & $\begin{array}{l}1880- \\
2015 \\
\end{array}$ & $\begin{array}{l}\text { Alaska } \\
\text { (Smith \& Herrero, 2018) }\end{array}$ & $N R$ & NR & NR & 289 & \begin{tabular}{|l}
289 \\
$(135$ years $)$ \\
\end{tabular} \\
\hline & $\begin{array}{l}1900- \\
2004 \\
\end{array}$ & $\begin{array}{l}\text { Alaska } \\
\text { (Smith et al., 2005) }\end{array}$ & NR & $\mathrm{NR}$ & $\mathrm{NR}$ & 420 & $\begin{array}{l}420 \\
\text { (104 years) }\end{array}$ \\
\hline & $\begin{array}{l}1977- \\
2016 \\
\end{array}$ & $\begin{array}{l}\text { Scandinavia } \\
\text { (Støen et al., 2018) }\end{array}$ & $N R$ & 26 & NR & 16 & $\begin{array}{l}42 \\
(39 \text { years }) \\
\end{array}$ \\
\hline \multirow[t]{2}{*}{$\begin{array}{l}\text { Sloth bear } \\
\text { (Melursus ursinus) }\end{array}$} & $\begin{array}{l}1938- \\
2004 \\
\end{array}$ & $\begin{array}{l}\text { Sri Lanka } \\
\text { (Ratnayeke et al., 2014) }\end{array}$ & NR & 90 & $N R$ & 181 & \begin{tabular}{|l}
271 \\
(66 years) \\
\end{tabular} \\
\hline & $\begin{array}{l}2003- \\
2013 \\
\end{array}$ & $\begin{array}{l}\text { Chitwan NP, Nepal } \\
\text { (Ruda et al., 2018) }\end{array}$ & $N R$ & $N R$ & NR & 59 & $\begin{array}{l}59 \\
(10 \text { years }) \\
\end{array}$ \\
\hline \multirow[t]{4}{*}{$\begin{array}{l}\text { Polar bear } \\
\text { (Ursus maritimus) }\end{array}$} & $\begin{array}{l}1900- \\
2000\end{array}$ & $\begin{array}{l}\text { United States, Norway } \\
\text { (Svalbard), \& Canada } \\
\text { (Löe \& Röskaft, 2004) } \\
\end{array}$ & NR & NR & $N R$ & 12 & $\begin{array}{l}12^{\star} \\
(100 \text { years })\end{array}$ \\
\hline & $\begin{array}{l}2005- \\
2016 \\
\end{array}$ & $\begin{array}{l}\text { Global } \\
\text { (Bombieri et al., 2018) }\end{array}$ & $N R$ & NR & NR & 68 & $\begin{array}{l}68 \\
(11 \text { years }) \\
\end{array}$ \\
\hline & $\begin{array}{l}1870- \\
2014 \\
\end{array}$ & $\begin{array}{l}\text { Global } \\
\text { (Wilder et al., 2017) }\end{array}$ & 37 & 30 & 0 & 6 & $\begin{array}{l}73 \\
\text { (144 years) } \\
\end{array}$ \\
\hline & $\begin{array}{l}1955- \\
2014\end{array}$ & $\begin{array}{l}\text { North America, Europe } \\
\text { (Svalbard, Norway), \& } \\
\text { Russia (Penteriani et al., } \\
\text { 2016) }\end{array}$ & $N R$ & NR & $N R$ & 13 & $\begin{array}{l}13 \\
(59 \text { years })\end{array}$ \\
\hline
\end{tabular}


Human Ecology Review, Volume 25, Number 2, 2019

\begin{tabular}{|c|c|c|c|c|c|c|c|}
\hline & $\begin{array}{l}\text { Date } \\
\text { range }\end{array}$ & Location (Source) & 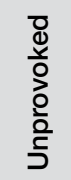 & 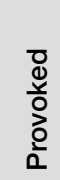 & 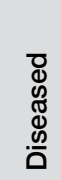 & 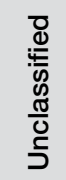 & $\begin{array}{l}\text { Total attacks } \\
\text { (per date range) }\end{array}$ \\
\hline \multicolumn{8}{|l|}{ Dogs (Canidae) } \\
\hline \multirow[t]{3}{*}{$\begin{array}{l}\text { Coyote } \\
\text { (Canis latrans) }\end{array}$} & $\begin{array}{l}1960- \\
2006\end{array}$ & $\begin{array}{l}\text { Urban North America } \\
\text { (White \& Gehrt, 2009) }\end{array}$ & 95 & 13 & 15 & 36 & $\begin{array}{l}159^{\star \star} \\
(46 \text { years) }\end{array}$ \\
\hline & $\begin{array}{l}1960- \\
1988\end{array}$ & $\begin{array}{l}\text { Western United States } \\
\text { \& Canada } \\
\text { (Carbyn, 1989) }\end{array}$ & 4 & NR & NR & 10 & $\begin{array}{l}14 \\
\text { (28 years) }\end{array}$ \\
\hline & $\begin{array}{l}1978- \\
2003\end{array}$ & $\begin{array}{l}\text { California } \\
\text { (Timm et al., 2004) }\end{array}$ & NR & NR & NR & 89 & $\begin{array}{l}89 \\
(25 \text { years })\end{array}$ \\
\hline \multirow[t]{3}{*}{$\begin{array}{l}\text { Wolf } \\
\text { (Canis lupus) }\end{array}$} & $\begin{array}{l}1900- \\
2000 \\
\end{array}$ & \begin{tabular}{|l} 
Global\#\# \\
(Löe \& Röskaft, 2004) \\
\end{tabular} & $N R$ & NR & $N R$ & 607 & $\begin{array}{l}607 \\
(100 \text { years }) \\
\end{array}$ \\
\hline & $\begin{array}{l}2001- \\
2012\end{array}$ & $\begin{array}{l}\text { Hamedan Province, Iran } \\
\text { (Behdarvand \& Kaboli, } \\
\text { 2015) }\end{array}$ & 36 & 12 & 0 & 5 & $\begin{array}{l}53 \\
(11 \text { years })\end{array}$ \\
\hline & $\begin{array}{l}1969- \\
2000\end{array}$ & $\begin{array}{l}\text { United States (Alaska) } \\
\text { \& Canada } \\
\text { (McNay, 2002) }\end{array}$ & $18^{\wedge}$ & 20 & 12 & 0 & $\begin{array}{l}50 \\
\text { (31 years) }\end{array}$ \\
\hline $\begin{array}{l}\text { Spotted hyena } \\
\text { (Crocuta crocuta) }\end{array}$ & $\begin{array}{l}1923- \\
1994\end{array}$ & $\begin{array}{l}\text { Uganda } \\
\text { (Treves \& Naughton- } \\
\text { Treves, 1999) } \\
\end{array}$ & NR & NR & $N R$ & 4 & $\begin{array}{l}4 \\
\text { (71 years) }\end{array}$ \\
\hline $\begin{array}{l}\text { Striped hyena } \\
\text { (Hyena hyena) }\end{array}$ & $\begin{array}{l}1900- \\
2000\end{array}$ & $\begin{array}{l}\text { India } \\
\text { (Löe \& Röskaft, 2004) }\end{array}$ & NR & NR & $N R$ & 2 & $\begin{array}{l}2 \\
\text { (100 years) }\end{array}$ \\
\hline \multicolumn{8}{|l|}{ Cats (Felidae) } \\
\hline $\begin{array}{l}\text { Cheetah } \\
\text { (Acinonyx } \\
\text { jubatus) }\end{array}$ & $\begin{array}{l}1979- \\
2007\end{array}$ & $\begin{array}{l}\text { Global } \\
\text { (Inskip \& Zimmermann, } \\
\text { 2009) }\end{array}$ & 0 & 0 & 0 & 0 & $\begin{array}{l}0 \\
(28 \text { years })\end{array}$ \\
\hline \multirow[t]{4}{*}{$\begin{array}{l}\text { Cougar } \\
\text { (Puma concolor) }\end{array}$} & $\begin{array}{l}1900- \\
2000\end{array}$ & $\begin{array}{l}\text { Chile, Canada, \& United } \\
\text { States } \\
\text { (Löe \& Röskaft, 2004) }\end{array}$ & $N R$ & $N R$ & $N R$ & 18 & $\begin{array}{l}18 \\
\text { (100 years) }\end{array}$ \\
\hline & $\begin{array}{l}1980- \\
2016\end{array}$ & $\begin{array}{l}\text { Urban United States } \\
\text { \& Canada } \\
\text { (Bombieri et al., 2018) }\end{array}$ & 12 & 0 & 0 & 0 & $\begin{array}{l}12 \\
(36 \text { years) }\end{array}$ \\
\hline & $\begin{array}{l}1890- \\
1990 \\
\end{array}$ & $\begin{array}{l}\text { United States \& Canada } \\
\text { (Beier, 1991) }\end{array}$ & $N R$ & NR & NR & 53 & $\begin{array}{l}53 \\
\text { (100 years) }\end{array}$ \\
\hline & $\begin{array}{l}1890- \\
2008\end{array}$ & $\begin{array}{l}\text { United States \& Canada } \\
\text { (Mattson et al., 2011) }\end{array}$ & NR & NR & $N R$ & 200 & $\begin{array}{l}200 \\
(118 \text { years })\end{array}$ \\
\hline \multirow[t]{2}{*}{$\begin{array}{l}\text { Jaguar } \\
\text { (Panthera onca) }\end{array}$} & 1995 & $\begin{array}{l}\text { Argentina (North Chaco) } \\
\text { (Inskip \& Zimmermann, } \\
\text { 2009) }\end{array}$ & $N R$ & NR & $N R$ & 1 & $\begin{array}{l}1 \\
\text { (1 year) }\end{array}$ \\
\hline & $\begin{array}{l}1940- \\
2015\end{array}$ & $\begin{array}{l}\text { Venezuela } \\
\text { (Jędrzejewski et al., 2017) }\end{array}$ & $N R$ & 7 & $N R$ & 15 & $\begin{array}{l}22 \\
(75 \text { years })\end{array}$ \\
\hline
\end{tabular}




\begin{tabular}{|c|c|c|c|c|c|c|c|}
\hline & $\begin{array}{l}\text { Date } \\
\text { range }\end{array}$ & Location (Source) & 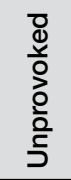 & 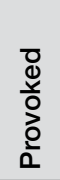 & 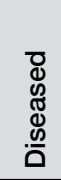 & 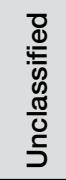 & $\begin{array}{l}\text { Total attacks } \\
\text { (per date range) }\end{array}$ \\
\hline \multirow[t]{7}{*}{$\begin{array}{l}\text { Leopard } \\
\text { (Panthera pardus) }\end{array}$} & $\begin{array}{l}1900- \\
2000\end{array}$ & $\begin{array}{l}\text { India, Nepal, Uganda, } \\
\text { South Africa } \\
\text { (Löe \& Röskaft, 2004) }\end{array}$ & NR & NR & NR & 840 & $\begin{array}{l}840^{*} \\
(100 \text { years) }\end{array}$ \\
\hline & $\begin{array}{l}2005- \\
2016\end{array}$ & $\begin{array}{l}\text { Global } \\
\text { (Bombieri et al., 2018) }\end{array}$ & $N R$ & $N R$ & NR & 177 & $\begin{array}{l}177 \\
(11 \text { years })\end{array}$ \\
\hline & $\begin{array}{l}2003- \\
2013\end{array}$ & $\begin{array}{l}\text { Chitwan NP, Nepal } \\
\text { (Ruda et al., 2018) }\end{array}$ & NR & $N R$ & NR & 18 & $\begin{array}{l}18 \\
(10 \text { years })\end{array}$ \\
\hline & $\begin{array}{l}2005- \\
2011\end{array}$ & $\begin{array}{l}\text { Central India } \\
\text { (Dhanwatey et al., 2013) }\end{array}$ & $N R$ & NR & NR & 29 & $\begin{array}{l}29 \\
(6 \text { years })\end{array}$ \\
\hline & $\begin{array}{l}1993- \\
2003 \\
\end{array}$ & $\begin{array}{l}\text { Maharashtra, India } \\
\text { (Packer et al., 2018) }\end{array}$ & $\mathrm{NR}$ & NR & NR & 67 & $\begin{array}{l}67 \\
(10 \text { years }) \\
\end{array}$ \\
\hline & $\begin{array}{l}2004- \\
2014 \\
\end{array}$ & $\begin{array}{l}\text { Himachal Pradesh, India } \\
\text { (Packer et al., 2018) }\end{array}$ & NR & NR & NR & 329 & $\begin{array}{l}329 \\
(10 \text { years })\end{array}$ \\
\hline & $\begin{array}{l}1923- \\
1994\end{array}$ & $\begin{array}{l}\text { Uganda } \\
\text { (Treves \& Naughton- } \\
\text { Treves, 1999) }\end{array}$ & NR & 4 & NR & 110 & $\begin{array}{l}114 \\
\text { (71 years) }\end{array}$ \\
\hline \multirow[t]{8}{*}{$\begin{array}{l}\text { Lion } \\
\text { (Panthera leo) }\end{array}$} & $\begin{array}{l}1900- \\
2000\end{array}$ & $\begin{array}{l}\text { India, South Africa, } \\
\text { Tanzania, Uganda, Zambia } \\
\text { (Löe \& Röskaft, 2004) }\end{array}$ & NR & NR & NR & 552 & $\begin{array}{l}552^{*} \\
(100 \text { years) }\end{array}$ \\
\hline & $\begin{array}{l}1990- \\
2007\end{array}$ & $\begin{array}{l}\text { Rufiji \& Lindi, Tanzania } \\
\text { (Kushnir, 2009) }\end{array}$ & 274 & 0 & 0 & 0 & $\begin{array}{l}274 \\
\text { (17 years) }\end{array}$ \\
\hline & $\begin{array}{l}1989- \\
2008 \\
\end{array}$ & $\begin{array}{l}\text { Tanzania } \\
\text { (Packer et al., 2018) }\end{array}$ & $\mathrm{NR}$ & $\mathrm{NR}$ & NR & 319 & $\begin{array}{l}319 \\
(19 \text { years }) \\
\end{array}$ \\
\hline & $\begin{array}{l}2005- \\
2016 \\
\end{array}$ & $\begin{array}{l}\text { Global } \\
\text { (Bombieri et al., 2018) }\end{array}$ & NR & NR & NR & 73 & $\begin{array}{l}73 \\
(11 \text { years }) \\
\end{array}$ \\
\hline & $\begin{array}{l}1923- \\
1994\end{array}$ & $\begin{array}{l}\text { Uganda } \\
\text { (Treves \& Naughton- } \\
\text { Treves, 1999) }\end{array}$ & NR & 5 & $N R$ & 270 & $\begin{array}{l}275 \\
\text { (71 years) }\end{array}$ \\
\hline & $\begin{array}{l}2006- \\
2008\end{array}$ & $\begin{array}{l}\text { Mozambique } \\
\text { (Dunham et al., 2010) }\end{array}$ & NR & NR & NR & 44 & $\begin{array}{l}44^{\star \star} \\
(2 \text { years })\end{array}$ \\
\hline & $\begin{array}{l}2006- \\
2010 \\
\end{array}$ & $\begin{array}{l}\text { Mozambique } \\
\text { (Le Bel et al., 2011) }\end{array}$ & NR & NR & NR & 17 & $\begin{array}{l}17 \\
\text { (4 years) } \\
\end{array}$ \\
\hline & $\begin{array}{l}2002- \\
2008 \\
\end{array}$ & $\begin{array}{l}\text { Zambia } \\
\text { (Chomba et al., 2012) }\end{array}$ & NR & NR & NR & 21 & $\begin{array}{l}21 \\
\text { (6 years) }\end{array}$ \\
\hline $\begin{array}{l}\text { Snow leopard } \\
\text { (Panthera uncia) }\end{array}$ & $\begin{array}{l}1979- \\
2007\end{array}$ & $\begin{array}{l}\text { Global } \\
\text { (Inskip \& Zimmermann, } \\
\text { 2009) }\end{array}$ & 0 & 0 & 0 & 0 & $\begin{array}{l}0 \\
\text { (28 years) }\end{array}$ \\
\hline
\end{tabular}




\begin{tabular}{|c|c|c|c|c|c|c|c|}
\hline & $\begin{array}{l}\text { Date } \\
\text { range }\end{array}$ & Location (Source) & 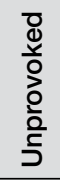 & 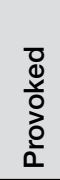 & 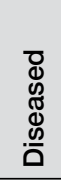 & 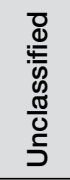 & $\begin{array}{l}\text { Total attacks } \\
\text { (per date range) }\end{array}$ \\
\hline \multirow[t]{6}{*}{$\begin{array}{l}\text { Tiger } \\
\text { (Panthera tigris) }\end{array}$} & $\begin{array}{l}2003- \\
2013 \\
\end{array}$ & $\begin{array}{l}\text { Chitwan NP, Nepal } \\
\text { (Ruda et al., 2018) }\end{array}$ & NR & $N R$ & NR & 68 & $\begin{array}{l}68 \\
(10 \text { years }) \\
\end{array}$ \\
\hline & $\begin{array}{l}1979- \\
2006\end{array}$ & $\begin{array}{l}\text { Chitwan NP, Nepal } \\
\text { (Packer et al., 2018) }\end{array}$ & NR & NR & NR & 88 & $\begin{array}{l}88 \\
\text { (27 years) }\end{array}$ \\
\hline & $\begin{array}{l}2005- \\
2016 \\
\end{array}$ & $\begin{array}{l}\text { Global } \\
\text { (Bombieri, 2018) }\end{array}$ & NR & NR & NR & 31 & $\begin{array}{l}31 \\
\text { (11 years) }\end{array}$ \\
\hline & $\begin{array}{l}2005- \\
2011 \\
\end{array}$ & \begin{tabular}{|l} 
India \\
(Dhanwatey et al., 2013) \\
\end{tabular} & NR & NR & NR & 103 & $\begin{array}{l}103 \\
\text { (6 years) } \\
\end{array}$ \\
\hline & $\begin{array}{l}2005- \\
2010 \\
\end{array}$ & $\begin{array}{l}\text { Maharashtra, India } \\
\text { (Packer et al., 2018) }\end{array}$ & NR & NR & NR & 94 & $\begin{array}{l}94 \\
\text { (5 years) } \\
\end{array}$ \\
\hline & $\begin{array}{l}1979- \\
2006\end{array}$ & $\begin{array}{l}\text { Chitwan NP, Nepal } \\
\text { (Packer et al., 2018) }\end{array}$ & $N R$ & NR & NR & 88 & $\begin{array}{l}88 \\
\text { (27 years) }\end{array}$ \\
\hline \multicolumn{8}{|l|}{ Sharks } \\
\hline \multirow[t]{3}{*}{ Shark } & $\begin{array}{l}2007- \\
2018 \\
\end{array}$ & $\begin{array}{l}\text { Global } \\
\text { (ISAF, 2018) }\end{array}$ & NR & NR & NR & 984 & $\begin{array}{l}984 \\
\text { (11 years) } \\
\end{array}$ \\
\hline & 2018 & $\begin{array}{l}\text { Global } \\
\text { (Naylor \& Bowling, 2018) }\end{array}$ & 66 & 34 & 0 & 30 & $\begin{array}{l}130 \\
\text { (1 year) }\end{array}$ \\
\hline & $\begin{array}{l}1950- \\
2003\end{array}$ & $\begin{array}{l}\text { United States (California } \\
\text { \& Oregon) } \\
\text { (McCosker \& Lea, 2006) }\end{array}$ & NR & NR & NR & 95 & $\begin{array}{l}95 \wedge \wedge \\
(53 \text { years })\end{array}$ \\
\hline
\end{tabular}

$\mathrm{NR}=$ not reported.

* This data figure concerns fatalities only, not all attacks.

**This data figure concerns attack victims, not attack numbers.

\#Canada, China, Japan, Yugoslavia, Kazakhstan, Kyrgyzstan, Mongolia, Norway, Romania, Russia, Sweden, and United States.

\#\#Afghanistan, Canada, China, Estonia, France, India, Iran, Italy, Latvia, Lithuania, Poland, Russia, Slovakia, Spain, and United States.

$\wedge$ Three of these cases were located in Minnesota, US, close to the Canadian border.

$\wedge \wedge$ This study data concerns only great white sharks (Carcharodon carcharias).

\section{Discussion}

\section{Coexisting with large carnivores}

Humans' actual relations with wild creatures exist in a perceived relationship informed by local cultural values and stories, personal histories, economic and technological empowerment, education and biases. More accurate reporting of carnivore attacks can augment and influence the often ad hoc nature of local understandings. Many factors drive the perceptions of wild animal attacks as dread threats. However, there is also an emerging public sentiment across many 
societies toward peaceful coexistence with wild animals and humane management techniques. Information from various regional studies can provide insights about practices applicable in compatible contexts. For example, a study in 2014 replicated a 1995 survey of public attitudes in the United States toward wildlife management, and found an increase in support for humane forms of predator management practices such as scare devices, fertility control, and non-lethal guard animals (Slagle et al., 2017, p. 13). Such results reflect a call for new and innovative practices for preventing human conflicts with large carnivores.

Aversive conditioning is an emerging approach toward peaceful coexistence with large carnivores and viewed as both safe and humane (Meadows \& Knowlton, 2000). Aversive conditioning, "an operant technique that uses a negative stimulus to cause pain, avoidance, or irritation in an animal engaged in an unwanted behavior" (Mazur, 2010, p. 48), can take different forms such as rubber bullets, loud noises, and dogs — with the goal of altering existing habituation and keeping wild animals away from humans in areas where interactions can be problematic (Mazur, 2010). The use of domesticated animals as guards is a common technique to ward off large carnivores. For example, guard dogs are employed to keep wolves away from livestock (Cluff \& Murray, 1995), llamas to protect sheep from coyotes (Meadows \& Knowlton, 2000), and Karelian bear dogs to scare away habituated bears. These methods are growing in popularity and are reported to be extremely effective (Crugnale et al., 2019). Such examples are promising non-lethal options, and more humane alternatives to other non-lethal methods such as relocation of human-conditioned large carnivores, a method that is problematic and ineffective (Crugnale et al., 2019; Isasi-Catalá, 2010).

In part, coexisting with wild animals means humans must take responsibility to avoid attacks. Given children are at a higher risk of being attacked (Bombieri et al., 2018), one strategy toward coexistence is to employ close supervision in regions where habituated large carnivores live and are known to be active. For adult humans, unprovoked attacks can be avoided as well, but interactions and encounters are contingent on cultural and physical conditions. For example, in the Western world, people may need to take extra caution when running, hiking, camping, or cycling in habitats where carnivores are active, or instead choose to do these recreational activities on trails where large carnivores are not as active. In their research on coyotes within the United States and Canada, White and Gehrt (2009) recommend sleeping in closed tents and paying careful attention when walking with a companion animal. In developing nations, and especially for rural communities, different considerations are foremost: firewood, water, and animal husbandry practices require travel into carnivore habitat, and farming practices may leave people more vulnerable to attack. For example, in rural Tanzania, lion attacks on humans are more common at harvest time because farmers sleep in the 
field to control the presence of bush pigs. Coexistence here requires controlling the numbers of bush pigs so that lions are not drawn to the area where farmers are (Packer et al., 2005).

Intentional provocations can, of course, always be avoided. For example, "attempts to ... kill the animal," "[luring] an animal closer to themselves" (Hockings et al., 2010 , p. 888), or "cornering [a] coyote" (White \& Gehrt, 2009, p. 423) are all instances where the human is responsible for the attack.

\section{Systematic records}

Similar to Löe and Röskaft (2004), we recommend more systematic record-keeping of large carnivore attacks. This will minimize inconsistencies among databases and government reports. For instance, the data included in Table 2 do not feature attack statistics from consistent timelines, regions, or sources. In creating systematic records of sightings and attacks we urge scholars, management experts, and communities to adopt uniform terminology. If attacks were reported under the definitions of Provoked and Unprovoked (e.g., Quigley \& Herrero, 2005), such consistency would allow for more definitive analyses of and insight into the drivers of human conflicts with large carnivores, and would in turn support improved management decisions. Similarly, if these terms entered the public and media discourse, the public would also be enabled to make better decisions for their safety, as well as gaining greater understanding, and possibly appreciation, of wild carnivores.

Several reports did thorough searches of a combination of government documents, academic literature, media reports, and databases (Mattson et al., 2011; McNay, 2002; White \& Gehrt, 2009), but many others drew from a narrower range of sources that would have excluded some reports. In addition to an exhaustive academic search, we recommend scholars include available gray literature when documenting attack information. Such efforts will allow for the most accurate and conclusive reporting.

Finally, specific details of each attack are necessary but are not common in the literature. For example, some sources did not report where the attack resulted in a fatality, or whether human provocation was a factor (Beier, 1991; Bombieri et al., 2018). Regarding coyotes, White and Gehrt (2009) found $47 \%$ of all victims were attacked while performing a recreational activity (e.g., jogging or hiking), and $19 \%$ while resting or sleeping outside. The activities people are engaged in prior to an attack and their behavior during an attack are also important, as is information on habituation patterns, and on intentional and unintentional provocations. Additionally, poor health in an animal should be investigated beyond disease, to include considerations for injury (either natural or human-induced), malnourishment, or other factors that may be causing unusual aggression. Precise information on the locations of attacks, including proximity to human settlements 
and even urban centers, will shed light on habituation patterns of species. Details of multiple attacks attributed to an individual animal should also be recorded and verified, where possible.

One way to include more information in record-keeping is to allow citizens to file attack reports online, where access to the internet is feasible. It is vital the communities who live among such species know how to report sightings. An inclusive approach for reporting would disseminate information (including how to report an attack and sightings) to all community members living in a shared ecological space with a large carnivore. White and Gehrt (2009) suggest recorded attack details for coyotes should include victim demographics, activities of the victim prior to the attack, coyote behavior prior to the attack, victim behavior during the attack, and presence of any companion animals in order to draw stronger conclusions about the nature of the attack. Further, we argue that photos to accurately identify species and individual animals are necessary for verifying sightings and gaining knowledge of habituation patterns. Löe and Röskaft (2004, p. 286) provide a table of 18 criteria used in various databases to describe encounters with or attacks on humans by large carnivores. Once a sighting is reported, appropriate information should be provided to the community, including how to avoid encounters and peacefully coexist with the large carnivore, as an educational and outreach response. Where possible, online reporting and email-generated responses with educational information could be provided.

Where online reporting is not feasible, we urge the development of methods suitable for the given community for gathering data and disseminating information. These methods could include appropriately trained workers or volunteers taking records of verbal reports, using techniques such as questionnaires addressing standardized criteria, maps for information on animal range, and species and individual identification photos.

\section{Risk from attacks}

From an empirical perspective, the actual risk of a large carnivore attack on a human is low when compared to other natural threats. For example, from 2009 to 2018, an average of 27 people died and 243 were injured annually from being struck by lightning in the United States alone (National Weather Service, 2019). In Canada and the United States combined, during a 32-year period from 1969 to 2000, 50 people were attacked by a wolf (McNay, 2002) — an average of less than two attacks per year. Similarly, during a 59-year time span from 1955 to 2014, there were 92 incidents of brown bear attacks-also an average of less than two per year (Penteriani et al., 2016). While it is difficult to record the number of people killed by lightning globally, estimates range between 6,000 and 24,000 per year (Perraudin, 2014). In 2018 there were 66 Unprovoked shark bites globally, resulting in four fatalities (van Hoose, 2019), a small number in comparison to the lowest estimation 
from lightning deaths. Such comparisons illuminate the low risk involved with human fatality related to large carnivores when placed next to natural accidents, of which lightning strike is only one example.

We have compared these data to highlight how misconceptions of the actual risk posed by large carnivores to humans differ in magnitude from the media portrayal of such incidents in the developed world (McCagh, 2015) and the storytelling that often occurs in the developing world that leads to "man-eater" constructions (Kelly, 2019). Management decisions should be based on accurate records that reflect reality, as opposed to basing them on public perceptions. Disentangling actual risk from perceptions of the threat is one avenue for future research that would illuminate misinformation and combat retaliatory responses to large carnivore attacks. If the ultimate goal of peaceful coexistence between humans and large carnivores is to be attained, we must understand the reality of such interactions, in number, in magnitude, and in nature.

\section{References}

Behdarvand, N., \& Kaboli, M. (2015). Characteristics of gray wolf attacks on humans in an altered landscape in the west of Iran. Human Dimensions of Wildlife, 20(2), 112-122. doi.org/10.1080/10871209.2015.963747

Beier, P. (1991). Cougar attacks on humans in the United States and Canada. Wildlife Society Bulletin (1973-2006), 19(4), 403-412.

Bombieri, G., Nanni, V., Delgado, M., Fedriani, J., López-Bao, J., Pedrini, P., \& Penteriani, V. (2018). Content analysis of media reports on predator attacks on humans: Toward an understanding of human risk perception and predator acceptance. BioScience, 68(8), 577-584. doi.org/10.1093/biosci/biy072

Carbyn, L. (1989). Coyote attacks on children in western North America. Wildlife Society Bulletin (1973-2006), 17(4), 444-446.

Chomba, C., Senzota, R., Chabwela, H., Mwitwa, J., \& Nyirenda, V. (2012). Patterns of human-wildlife conflicts in Zambia, causes, consequences and management responses. Journal of Ecology and the Natural Environment, 4(12), 303-313. doi.org/10.5897/ jene12.029

Cluff, H. D., \& Murray, D. L. (1995). Review of wolf control methods in North America. Ecology and Conservation of Wolves in a Changing World, 35, 491-607.

Crugnale, J., Humphrey, J. T., \& Reich, D. (2019). These dogs scare bears away to protect them. Retrieved September 2019, from nationalgeographic.com/animals/2019/02/ karelian-bear-dogs-keep-bears-away/ 
Dhanwatey, H., Crawford, J., Abade, L., Dhanwatey, P., Nielsen, C., \& Sillero-Zubiri, C. (2013). Large carnivore attacks on humans in Central India: A case study from the TadobaAndhari Tiger Reserve. Oryx, 47(2), 221-227. doi.org/10.1017/s0030605311001803

Dunham, K., Ghiurghi, A., Cumbi, R., \& Urbano, F. (2010). Human-wildlife conflict in Mozambique: A national perspective, with emphasis on wildlife attacks on humans. Oryx, 44(2), 185-193. doi.org/10.1017/s003060530999086x

Herrero, S., \& Higgins, A. (1999). Human injuries inflicted by bears in British Columbia: 1960-97. Ursus, 11, 209-218.

Herrero, S., Higgins, A., Cardoza, J., Hajduk, L., \& Smith, T. (2011). Fatal attacks by American black bear on people: 1900-2009. Journal of Wildlife Management, 75(3), 596-603. doi.org/10.1002/jwmg.72

Hockings, K., Yamakoshi, G., Kabasawa, A., Matsuzawa, T., \& Fuentes, A. (2010). Attacks on local persons by chimpanzees in Bossou, Republic of Guinea: Long-term perspectives. American Journal of Primatology, 72(10), 887-896. doi.org/10.1002/ajp.20784

Inskip, C., \& Zimmermann, A. (2009). Human-felid conflict: A review of patterns and priorities worldwide. Oryx, 43(1), 18-34. doi.org/10.1017/s003060530899030x

ISAF (International Shark Attack File) (2018). World locations with highest attack rates 2007-2016 [Data set]. floridamuseum.ufl.edu/shark-attacks/trends/location/world/

Isasi-Catalá, E. (2010). Is translocation of problematic jaguars (Panthera onca) an effective strategy to resolve human-predator conflicts? CEE Review 08-018 (SR55). Collaboration for Environmental Evidence. environmentalevidence.org/wp-content/uploads/2014/09/ SR55.pdf

Jędrzejewski, W., Carreño, R., Sánchez-Mercado, A., Schmidt, K., Abarca, M., Robinson, H. S., Boede, E. O., Hoogesteijn, R., Viloria, Á. L., Cerda, H., \& Velásquez, G. (2017). Human-jaguar conflicts and the relative importance of retaliatory killing and hunting for jaguar (Panthera onca) populations in Venezuela. Biological Conservation, 209, 524-532. doi.org/10.1016/j.biocon.2017.03.025

Kelly, J. R. (2019). A sociocultural perspective: Human conflict with jaguars and pumas in Costa Rica. Conservation and Society, 17(4), 355-365. doi.org/10.4103/cs.cs_17_141

Kushnir, H. (2009). Lion attacks on humans in southeastern Tanzania: Risk factors and perceptions (Doctoral dissertation). University of Minnesota, Minneapolis. ucl.ac.uk/pima/docs/ reference/04_lion_attacks.pdf

Le Bel, S., Murwira, A., Mukamuri, B., Czudek, R., Taylor, R., \& La Grange, M. (2011). Human wildlife conflicts in southern Africa: Riding the whirl wind in Mozambique and in Zimbabwe. In J. López-Pujol (Ed.), The importance of biological interactions in the study of biodiversity (pp. 283-322). IntechOpen. doi.org/10.5772/23682

Löe, J., \& Röskaft, E. (2004). Large carnivores and human safety: A review. Ambio, 33(6), 283-288. doi.org/10.1639/0044-7447(2004)033\%5b0283:Icahsa\%5d2.0.co;2 
Mattson, D., Logan, K., \& Sweanor, L. (2011). Factors governing risk of cougar attacks on humans. Human-Wildlife Interactions, 5(1), 135-158.

Mazur, R. (2010). Does aversive conditioning reduce human-black bear conflict? Journal of Wildlife Management, 74(1), 48-54. doi.org/10.2193/2008-163

McCagh, C., Sneddon, J., \& Blache, D. (2015). Killing sharks: The media's role in public and political response to fatal human-shark interactions. Marine Policy, 62, 271-278. doi.org/10.1016/j.marpol.2015.09.016

McCosker, J. E., \& Lea, R. N. (2006). White shark attacks upon humans in California and Oregon, 1993-2003. Proceedings of the California Academy of Science, 57(17), 479-501. calacademy.org/sites/default/files/assets/docs/pdf/mccoskerlea_5717_479-501_lr.pdf

McNay, M. E. (2002). Wolf-human interactions in Alaska and Canada: A review of the case history. Wildlife Society Bulletin, 30(3), 831-843.

Meadows, L., \& Knowlton, F. (2000). Efficacy of guard llamas to reduce canine predation on domestic sheep. Wildlife Society Bulletin, 28(3), 614-622.

National Weather Service (2019). How dangerous is lightning? [Data set]. Retrieved September 2019, from weather.gov/safety/lightning-odds

Naylor, G., \& Bowling, T. (2018). Yearly worldwide shark attack summary [Report]. International Shark Attack File. Florida Museum. floridamuseum.ufl.edu/shark-attacks/ yearly-worldwide-summary/

Packer, C., Ikanda, D., Kissui, B., \& Kushnir, H. (2005). Lion attacks on humans in Tanzania. Nature, 436(7053), 927-928. doi.org/10.1038/436927a

Packer, C., Shivakumar, S., Athreya, V., Craft, M. E., Dhanwatey, H., Dhanwatey, P., Gurung, B., Joshi, A., Kushnir, H., Linnell, J. D., \& Fountain-Jones, N. M. (2018). Species-specific spatiotemporal patterns of leopard, lion and tiger attacks on humans. Journal of Applied Ecology, 56(3), 585-593. doi.org/10.1111/1365-2664.13311

Penteriani, V., del Mar Delgado, M., Pinchera, F., Naves, J., Fernández-Gil, A., Kojola, I., Härkönen, S., Norberg, H., Frank, J., Fedriani, J. M., \& Sahlén, V., Støen, O-G., Swenson, J. E., Wabakken, P., Pellegrini, M., Herrero, S., \& López-Bao, J. V. (2016). Human behaviour can trigger large carnivore attacks in developed countries. Scientific Reports, 6(1), 1-12. doi.org/10.1038/srep20552

Perraudin, F., (2014, July 28). How many people are killed by lightning? The Guardian. theguardian.com/world/2014/jul/28/venice-beach-lightning-death-15th-this-year

Quigley, H., \& Herrero, S. (2005). Characterization and prevention of attacks on humans. In R. Woodroffe, S. J. Thirgood, \& A. Rabonowitz (Eds.), People and wildlife, conflict or co-existence? (pp. 27-48). Cambridge University Press. doi.org/10.1017/ cbo9780511614774.004 
Ratnayeke, S., Manen, F., Pieris, T., \& Pragash, R. (2014). Challenges of large carnivore conservation: Sloth bear attacks in Sri Lanka. Human Ecology, 42(3), 467-479. doi.org/ 10.1007/s10745-014-9643-y

Ruda, A., Kolejka, J., \& Silwal, T. (2018). GIS-assisted prediction and risk zonation of wildlife attacks in the Chitwan National Park in Nepal. ISPRS International Journal of Geo-Information, 7(9), 369. doi.org/10.3390/ijgi7090369

Slagle, K., Bruskotter, J., Singh, A., \& Schmidt, R. (2017). Attitudes toward predator control in the United States: 1995 and 2014. Journal of Mammalogy, 98(1), 7-16. doi.org/ 10.1093/jmammal/gyw144

Smith, T., Herrero, S., \& DeBruyn, T. (2005). Alaskan brown bears, humans, and habituation. Ursus, 16(1), 1-10. doi.org/10.2192/1537-6176(2005)016\%5b0001:abb hah\% $\% 5 \mathrm{~d} 2.0 . \mathrm{co} ; 2$

Smith, T., \& Herrero, S. (2018). Human-bear conflict in Alaska: 1880-2015. Wildlife Society Bulletin, 42(2), 254-263. doi.org/10.1002/wsb.870

Støen, O. G., Ordiz, A., Sahlén, V., Arnemo, J. M., Sæbø, S., Mattsing, G., Kristofferson, M., Brunberg, S., Kindberg, J., \& Swenson, J. E., (2018). Brown bear (Ursus arctos) attacks resulting in human casualties in Scandinavia 1977-2016: Management implications and recommendations. PloS ONE, 13(5), e0196876. doi.org/10.1371/journal.pone.0196876

Timm, R. M., Baker, R. O., Bennett, J. R., \& Coolahan, C. C. (2004). Coyote attacks: An increasing suburban problem. University of California Davis: Hopland Research and Extension Center. escholarship.org/uc/item/8qg662fb.

Treves, A., \& Naughton-Treves, L. (1999). Risk and opportunity for humans coexisting with large carnivores. Journal of Human Evolution, 36(3), 275-282. doi.org/10.1006/ jhev. 1998.0268

van Hoose, N. (2017). Global shark attacks drop to annual average in 2016. Florida Museum. floridamuseum.ufl.edu/science/global-shark-attacks-drop-to-annual-average-in-2016/

van Hoose, N. (2019). Shark bites plummet, fatalities remain average in 2018. Florida Museum. floridamuseum.ufl.edu/science/shark-bites-plummet-in-2018/

White, L., \& Gehrt, S. (2009). Coyote attacks on humans in the United States and Canada. Human Dimensions of Wildlife, 14(6), 419-432. doi.org/10.1080/10871200903055326

Wilder, J. M., Vongraven, D., Atwood, T., Hansen, B., Jessen, A., Kochnev, A., York, G., Vallender, R., Hedman, D., \& Gibbons, M. (2017). Polar bear attacks on humans: Implications of a changing climate. Wildlife Society Bulletin, 41(3), 537-547. doi.org/ 10.1002/wsb.783 
This text is taken from Human Ecology Review, Volume 25, Number 2, 2019, published by ANU Press, The Australian National University, Canberra, Australia. doi.org/10.22459/HER.25.02.2019.03 